\title{
Synthesis and physical properties of arc melted NiMnSb
}

\author{
S. Gardelis \\ Institute of Electronic Structure and Laser, Foundation for Research and Technology-Hellas, P.O. Box 1527, \\ Vasilika Vouton, 71110 Heraklion, Greece and Department of Materials Science and Technology, \\ University of Crete, P.O. Box 2208, 71003 Heraklion, Greece \\ J. Androulakis \\ Institute of Electronic Structure and Laser, Foundation for Research and Technology-Hellas, P.O. Box 1527, \\ Vasilika Vouton, 71110 Heraklion, Greece \\ P. Migiakis \\ Institute of Electronic Structure and Laser, Foundation for Research and Technology-Hellas, P.O. Box 1527, \\ Vasilika Vouton, 71110 Heraklion, Greece and Department of Physics, University of Crete, P.O. Box \\ 2208, 71003 Heraklion, Greece \\ J. Giapintzakis ${ }^{\text {a) }}$ \\ Institute of Electronic Structure and Laser, Foundation for Research and Technology-Hellas, P.O. Box 1527, \\ Vasilika Vouton, 71110 Heraklion, Greece and Department of Materials Science and Technology, \\ University of Crete, P.O. Box 2208, 71003 Heraklion, Greece
}

S. K. Clowes, Y. Bugoslavsky, W. R. Branford, Y. Miyoshi, and L. F. Cohen Blackett Laboratory, Imperial College, Prince Consort Road, London SW7 2BZ, United Kingdom

(Received 22 October 2003; accepted 22 March 2004)

\begin{abstract}
Several polycrystalline samples of the half-Heusler alloy NiMnSb were grown by arc melting of stoichiometric and nonstoichiometric amounts of high-purity constituent elements. The structure and the phase-purity of the prepared samples were examined systematically by powder x-ray diffraction. The transport properties of the best sample, with saturation magnetization $M_{s}(5 \mathrm{~K})$ $=4 \mu_{B}$ /formula unit, were studied by measuring electrical resistivity, thermal conductivity, and thermopower. Features in both magnetic and transport data are consistent with NiMnSb being in a half-metallic state at low temperatures, i.e., the conduction electrons are fully spin polarized. However, point-contact Andreev reflection measurements on the same sample at $4.2 \mathrm{~K}$ demonstrate only $\sim 45 \%$ spin polarization. (C) 2004 American Institute of Physics. [DOI: 10.1063/1.1739293]
\end{abstract}

\section{INTRODUCTION}

In recent years a technology called spintronics has emerged, which offers the potential for advanced devices that will combine spin-dependent effects arising from the spin of the electron with standard microelectronics. The advantage of the electron spin carrying the information in semiconductor devices, instead of the conventional electron charge, is the possibility of nonvolatility, lower power consumption, increased data processing speed, and increased integration densities. ${ }^{1}$

The proposal by Datta and $\operatorname{Das}^{2}$ for a spin-based transistor has prompted a vigorous search for establishing electrical spin injection from metallic ferromagnetic films into semiconductors. Recently, electrical spin injection from ferromagnetic permalloy thin films into a two-dimensional electron gas semiconductor system has been demonstrated. ${ }^{3}$ The observed effect was, however, less than $1 \%$ of its optimum magnitude and persisted only up to $10 \mathrm{~K}$. The inefficiency of electrical spin injection has been attributed partly to the electrical conductivity mismatch of the ferromagnetic metal and the semiconductor ${ }^{4}$ and partly to the low spin polarization of

a) Author to whom correspondence should be addressed; electronic mail: giapintz@iesl.forth.gr the permalloy (only 35\%). It has been proposed that using materials with $100 \%$ spin polarization, e.g., half-metallic ferromagnets, as spin injectors may enhance significantly the efficiency of the spin injection. ${ }^{4}$

Band structure calculations predict that the ternary halfHeusler alloy NiMnSb should exhibit 100\% spin polarization at the Fermi level. ${ }^{5}$ Due to its high Curie temperature $\left(T_{C}\right.$ $=730 \mathrm{~K})$ and its close structural similarity to zinc blende semiconductors, $\mathrm{NiMnSb}$ is suitable to be used as a spin injection material in spintronics.

$\mathrm{NiMnSb}$ crystallizes in the $\mathrm{C} 1_{b}$ structure, ${ }^{5}$ which consists of four interpenetrating fcc lattices equally spaced along the [111] direction. In the ordered alloy Ni occupies the lattice site at 000, Mn occupies the site at 1/4 1/4 1/4, Sb occupies the site at $3 / 43 / 43 / 4$, and the site at $1 / 21 / 21 / 2$ is unoccupied. The magnetic properties are due to magnetic moments localized at the $\mathrm{Mn}$ atoms, which interact via itinerant electrons in the conduction band. ${ }^{6}$ It has been calculated that 9 out of total 22 valence electrons are located in spin-down bands, and thus the total spin magnetic moment of NiMnSb becomes exactly $4.0 \mu_{B} .^{7}$

A literature search reveals that only a limited number of studies have focused on the physical properties of bulk $\mathrm{NiMnSb}$ samples addressing the alleged half metallicity of this compound. ${ }^{8,9}$ In this article we report on the identifica- 
tion of synthesis/annealing conditions for the preparation of single-phase NiMnSb ingots using arc melting as well as on the structural, magnetic, and transport properties of these materials. Our measurements demonstrate that it is possible to produce good quality bulk NiMnSb using arc melting, which is a faster, and easier method compared to the other techniques commonly used to grow bulk NiMnSb such as rf melting ${ }^{10}$ and solid-state reaction. ${ }^{11}$

\section{EXPERIMENT}

Various samples of NiMnSb were grown by arc melting and subjected to different postannealing treatments in order to identify the optimum conditions that result to stoichiometric single-phase samples. $\mathrm{Ni}(99.999 \%$ pure $), \mathrm{Mn}$ (99.999\% pure), and $\mathrm{Sb}$ (99.995\% pure) powders were weighted in a glove box under an $\operatorname{Ar}(99.999 \%$ pure) atmosphere and mixed thoroughly using an agate mortar and pestle. The resulted powders were pressed into pellets of 15 $\mathrm{mm}$ in diameter. The pellets were then placed in a watercooled copper crucible and arc melted in Ar atmosphere several times in order to improve the uniformity of the samples. Specimens were synthesized using stoichiometric amounts of $\mathrm{Ni}, \mathrm{Mn}$, and $\mathrm{Sb}(1: 1: 1)$ as well as 3\% and 5\% excess of $\mathrm{Mn}$, and being subjected to postannealing treatment in vacuum. Prior to the annealing the specimens were wrapped with tantalum foil in order to avoid any undesired oxidation and were placed in quartz tubes that were pumped down to a pressure of $5 \times 10^{-6}$ mbar. The quartz tubes were hermetically sealed and placed in a box furnace at $750{ }^{\circ} \mathrm{C}$ for several hours.

Powder X-ray diffraction patterns were recorded using a Rigaku (RINT 2000) diffractometer with monochromatic $\mathrm{Cu} K \alpha_{1}$ radiation. dc magnetization measurements were performed in a commercial extraction magnetometer at temperatures ranging from 5 to $300 \mathrm{~K}$. The resistivity was measured with the standard ac four-probe method in the temperature range $5 \leqslant T \leqslant 275 \mathrm{~K}$. Simultaneous thermal conductivity and thermopower measurements were performed in the temperature range 70-320 K using the steady-state method. The samples were glued with GE varnish to a copper cold finger that served as a heat sink. A small homemade manganin resistor heater was attached to the free end of the samples that served as a heat source. The samples had rectangular parallelepiped shape with typical dimensions $4.5 \times 5.5 \times 1.5 \mathrm{~mm}^{3}$. Small temperature differences $(\sim 0.5 \mathrm{~K})$ across the samples were measured using two chromel-constantan (type-E) thermocouples attached to two well-separated positions along the sample. The systematic error of thermal conductivity $\kappa(T)$ due to the uncertainty in the measurement of the sample size and the distance between the thermocouples was estimated to be $8 \%-10 \%$. Seebeck voltages were detected using a pair of $0.05 \mathrm{~mm}$ diameter copper wires attached to the samples with silver paste at the same positions as the junctions of the thermocouples. The contribution of the copper leads to the measured Seebeck coefficient was carefully subtracted. Finally, an estimate of the transport spin polarization $\left(P_{t}\right)$ of the samples was obtained by point-contact Andreev reflection (PCAR) measurements ${ }^{12}$ using a mechanically formed superconducting $\mathrm{Nb}$ tip. Conductance $(d I / d V)$ spectra were
TABLE I. Growth conditions for several samples accompanied by the lattice constant and percentage of NiSb phase determined from analyzed powder $\mathrm{XRD}$ spectra as well as the saturation magnetic moment at RT and $5 \mathrm{~K}$ in some cases.

\begin{tabular}{|c|c|c|c|c|}
\hline $\begin{array}{l}\text { Specimen } \\
\text { No. }\end{array}$ & Growth conditions & $\begin{array}{l}\text { Lattice } \\
\text { constant } \\
(\AA)\end{array}$ & $\begin{array}{l}\mathrm{NiSb} \\
\text { phase } \\
(\%)\end{array}$ & $\begin{array}{l}\text { Magnetic } \\
\text { moment } \\
\left(\mu_{B}\right)\end{array}$ \\
\hline 1 & $\begin{array}{l}\text { Ni:Mn:Sb }(1: 1: 1) \text { arc } \\
\text { melted five times }\end{array}$ & 5.931 & 17 & 3.24 at $\mathrm{RT}$ \\
\hline 2 & $\begin{array}{l}\text { Annealing of sample } \\
\text { No. } 1 \text { in vacuum at } \\
750{ }^{\circ} \mathrm{C} \text { for } 50 \mathrm{~h}\end{array}$ & 5.927 & 5 & $\begin{array}{l}3.49 \text { at } \mathrm{RT} \\
3.68 \text { at } 5 \mathrm{~K}\end{array}$ \\
\hline 3 & $\begin{array}{l}\text { Further annealing of } \\
\text { sample No. } 2 \text { for } 72 \mathrm{~h} \\
\text { under the same } \\
\text { conditions }\end{array}$ & 5.926 & 11 & 3.18 at $\mathrm{RT}$ \\
\hline 4 & $\begin{array}{l}3 \% \text { Mn in excess, arc } \\
\text { melted five times }\end{array}$ & 5.936 & 3 & 3.5 at RT \\
\hline 5 & $\begin{array}{l}5 \% \mathrm{Mn} \text { in excess, arc } \\
\text { melted five times }\end{array}$ & 5.922 & 2 & 3.52 at $\mathrm{RT}$ \\
\hline 6 & $\begin{array}{l}\text { Annealing of sample } \\
\text { No. } 5 \text { in vacuum at } \\
7500^{\circ} \mathrm{C} \text { for } 50 \mathrm{~h}\end{array}$ & 5.921 & $<2$ & $\begin{array}{l}3.60 \text { at } \mathrm{RT} \\
4.02 \text { at } 5 \mathrm{~K}\end{array}$ \\
\hline
\end{tabular}

recorded using a standard four-point configuration with an ac lock-in amplifier.

\section{RESULTS AND DISCUSSION}

\section{A. Structure}

The powder x-ray diffraction (XRD) spectra of all investigated specimens exhibited mainly peaks corresponding to $\mathrm{NiMnSb}$ phase. However, an extra phase, which was always identified as NiSb, appeared in the XRD patterns of all specimens and its relative abundance depended on the molar ratio of the starting powders and the postannealing treatment. The XRD patterns were numerically analyzed $^{13}$ using a Thomson-Cox-Hastings pseudo-Voigt function to describe the peak shape. In this analysis, the NiSb phase was also taken into consideration in addition to the NiMnSb phase and the background was defined by linear interpolation through a number of selected background points. Table I summarizes the results obtained from several $\mathrm{NiMnSb}$ specimens that were prepared under different conditions.

Figure 1(a) shows the XRD pattern of sample No. 1 grown by arc-melting five consecutive times stoichiometric (1:1:1) amounts of $\mathrm{Ni}, \mathrm{Mn}$, and $\mathrm{Sb}$ powders. It can be seen that the NiSb-related peaks are pronounced in this case. Following annealing in vacuum at $750^{\circ} \mathrm{C}$ for $50 \mathrm{~h}$, the intensity of the NiSb peaks decreased considerably (sample No. 2 in Table I). Further annealing under the same conditions for an additional $22 \mathrm{~h}$ (total annealing of $72 \mathrm{~h}$ ) resulted in a reenhancement of the intensity of the NiSb peaks (sample No. 3 in Table I). Therefore, the annealing time seems to be a critical parameter in obtaining good quality materials.

In specimens grown with $3 \%$ excess $\mathrm{Mn}$, the percentage of the NiSb phase is reduced significantly to about $3 \%$ relative to the NiMnSb phase (sample No. 4 in Table I). Annealing of these specimens in vacuum at $750^{\circ} \mathrm{C}$ for $50 \mathrm{~h}$ although improved the magnetic properties of the material did 

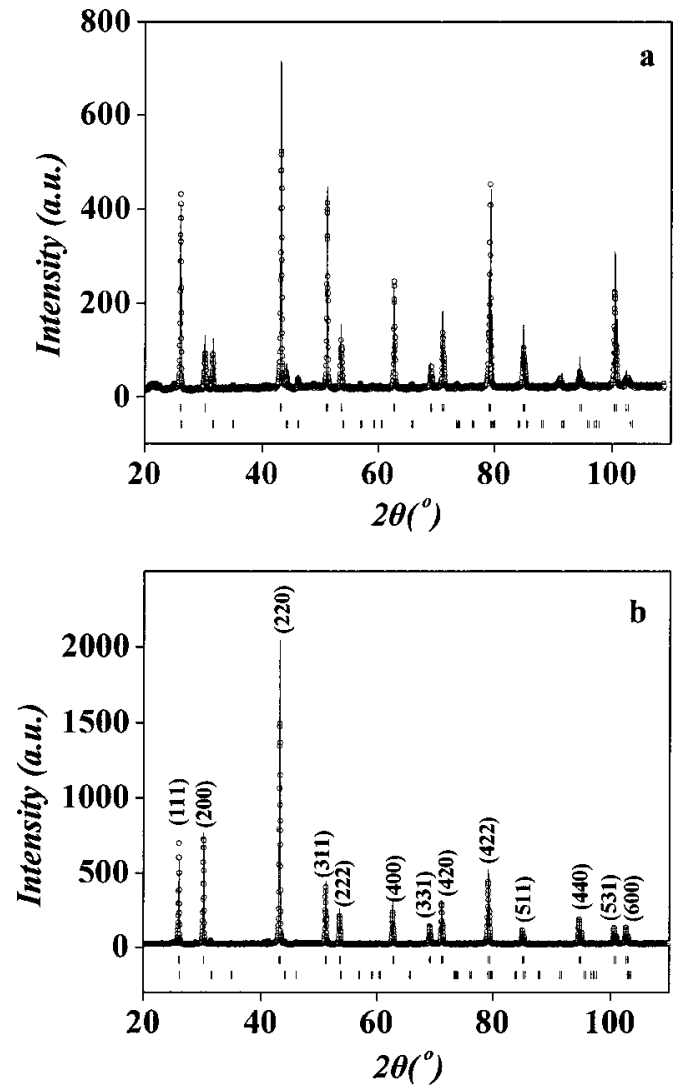

FIG. 1. X-ray diffraction pattern obtained from specimens: No. 1 (a) and No. 6 (b). In all graphs the first raw of vertical lines corresponds to peaks expected for $\mathrm{NiMnSb}$ and the second raw corresponds to peaks expected for $\mathrm{NiSb}$.

not result in any detectable change in the XRD patterns. On the other hand, sample No. 6 grown with 5\% excess Mn and annealed in vacuum for $50 \mathrm{~h}$ at $750{ }^{\circ} \mathrm{C}$ showed the best XRD spectrum [Fig. 1(b)], i.e., the NiSb phase was reduced to less than $2 \%$. The room-temperature lattice constant of the best sample No. 6 was measured to be 5.921(1) $\AA$, similar to the values reported previously by Szytuła et al. ${ }^{11}$ and Otto et al. ${ }^{6}$ The best samples are, thus, obtained using 5\% excess $\mathrm{Mn}$ in the initial powder mixture and postannealing the multiply arc-melted pellets in vacuum at $750{ }^{\circ} \mathrm{C}$ for $50 \mathrm{~h}$.

\section{B. Magnetic properties}

As can be seen from Table I, the specimen that shows the best XRD spectrum exhibits an integer saturation magnetic moment at $5 \mathrm{~K}\left[M_{s}(5 \mathrm{~K})=4.00 \pm 0.02 \mu_{B}\right.$ /f.u.; Fig. 2], as expected for a half-metallic ferromagnet. In general, it is found that the presence of the NiSb phase in the material leads to a rapid decrease of the value of $M_{s}$. The fact that we have to use at least 3\% excess $\mathrm{Mn}$ in order to concurrently decrease the amount of NiSb present in the sample and improve its magnetic properties, implies that Mn is most likely consumed faster than $\mathrm{Ni}$ and $\mathrm{Sb}$ during arc melting leading to the formation of the undesired $\mathrm{NiSb}$ phase.

Figure 3 depicts the temperature dependence of the saturation magnetization $M_{S}$ for specimen No. 6 obtained in a

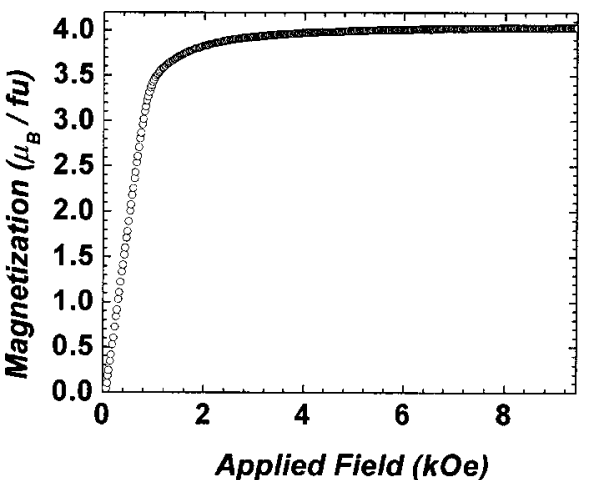

FIG. 2. Field dependence of the magnetization for specimen No. 6 at $5 \mathrm{~K}$.

magnetic field of $20 \mathrm{kOe}$. The magnetization data up to 200 $\mathrm{K}$ can be fitted well, as shown by the solid line in Fig. 3(a), to the Bloch law

$$
M_{S}=M_{S}(0)\left(1-A T^{3 / 2}\right),
$$

where $M_{S}(0)$ is the extrapolated saturation magnetization at $0 \mathrm{~K}$, and $A=1 \times 10^{-5} \mathrm{~K}^{-3 / 2}$. The same power-law temperature dependence of the magnetization has been observed previously, however, only up to $70 \mathrm{~K} .{ }^{8}$ It is noteworthy that the present magnetization data follow the Bloch $T^{3 / 2}$-law up to $200 \mathrm{~K}$; this is consistent with the fact that the Bloch law should hold only up to $T \sim T_{C} / 3$ (for NiMnSb, $T_{C}=720 \mathrm{~K}$ ). Hence, at low temperatures the system behaves like a Heisenberg ferromagnet as expected for a half-metal ferro-
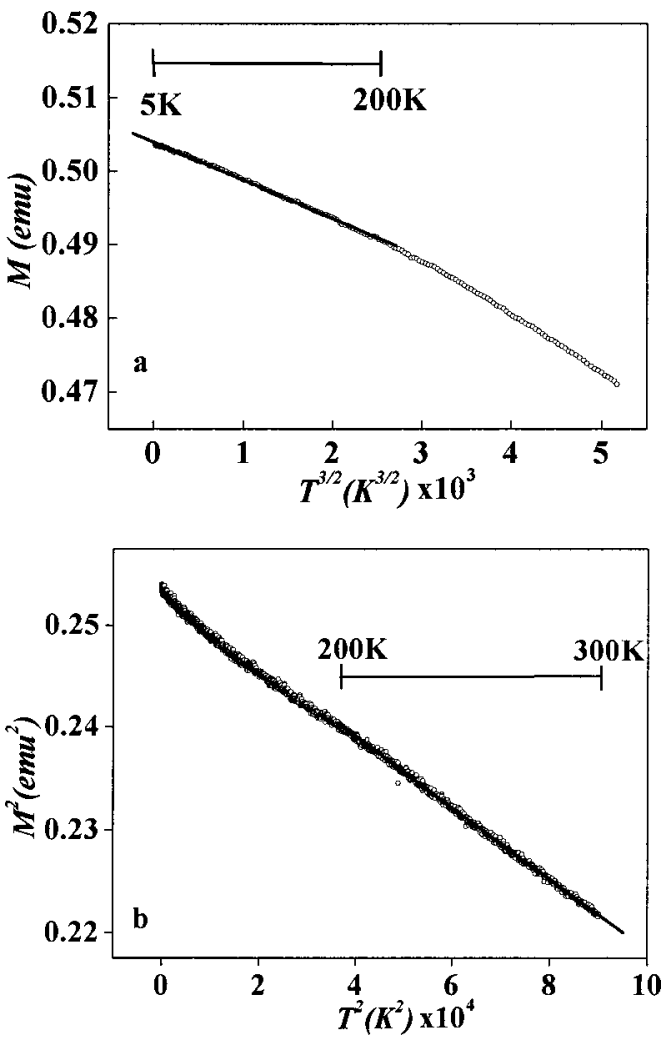

FIG. 3. Fit of the temperature dependence of the saturation magnetic moment $M_{s}$ (emu) for specimen No. 6 measured in an applied magnetic field of $20 \mathrm{kOe}$ in the temperature range: 5-200 (a) and 200-300 K (b). 


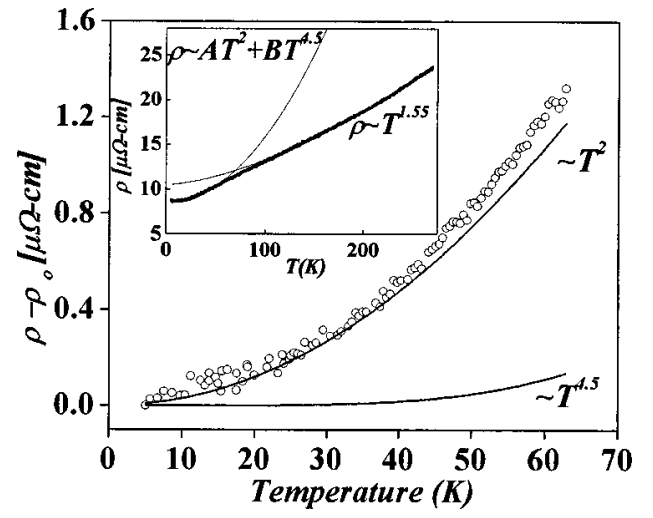

FIG. 4. Temperature dependence of the resistivity for specimen No. 6 in the temperature range $5-60 \mathrm{~K}$. The contributions of both $T^{2}$ and $T^{9 / 2}$ terms are shown. The inset shows fits of the resistivity vs temperature curve for the low- and high-temperature range.

magnet. Using the value of $A$ that resulted from the fitting procedure, the spin wave stiffness coefficient is calculated according to the following expression: ${ }^{14}$

$$
A=2.612(V / S)\left(k_{B} / 4 \pi D\right)^{3 / 2},
$$

where $V$ is the volume per magnetic atom, $S$ is the spin of the magnetic atom, and $k_{B}$ is the Boltzmann coefficient. Taking into account the fact that the magnetic moments are well localized at the Mn sites ${ }^{15}$ we have used as $V$ and $S$ the volume and the spin of the $\mathrm{Mn}$ atom, respectively. Thus, for $V=2.3 \times 10^{-24} \mathrm{~cm}^{3}$ and $S=5 / 2$, we find $D=270 \mathrm{meV} \AA^{2}$. This value for $D$ is close to values reported previously. For example, based on neutron diffraction data, $D$ was calculated to be $320 \pm 20 \mathrm{meV} \mathrm{A}^{2}$ at $25 \mathrm{~K} .{ }^{9}$ Hordequin et al. deduced $305 \pm 40 \mathrm{meV} \AA^{2}$ from the $T^{3 / 2}$ temperature dependence of the magnetization in the temperature range 4-70 $\mathrm{K},{ }^{9}$ whereas Otto et al. deduced a value of $350 \pm 40 \mathrm{meV} \mathrm{A}^{2}$ from the $T^{3 / 2}$ variation of the magnetization in the temperature range $4-300 \mathrm{~K}^{8}{ }^{8}$ For temperatures above $200 \mathrm{~K}$ [see Fig. 3(b)], a different $T$ dependence of the magnetization was found, $M_{S}^{2} \sim T^{2}$. It is noteworthy that such a power-law $T$ dependence of the magnetization has been predicted to occur in itinerant ferromagnets due to spin fluctuations. ${ }^{16}$ At these high temperatures both spin bands most likely contribute to transport and individual spin reversals can occur, i.e., Stoner excitations modify the temperature dependence of the magnetization. Therefore, the magnetization results are consistent with a crossover from a Heisenberg ferromagnet to an itinerant ferromagnet at $T \sim 200 \mathrm{~K}$.

\section{Transport properties}

\section{Resistivity}

The temperature dependence of the zero-field resistivity, $\rho(T)$, for specimen No. 6 in the temperature range $5 \leqslant T$ $\leqslant 275 \mathrm{~K}$ is shown in the inset of Fig. 4. A pronounced anomaly occurs in the $\rho(T)$ curve in the temperature range $70-120 \mathrm{~K}$. For temperatures below $70 \mathrm{~K}$, the resistivity has been fitted successfully to the expression

$$
\rho(T)=\rho_{0}+A T^{2}+B T^{9 / 2},
$$

with $\rho_{0}=8.6 \mu \Omega \mathrm{cm}, \quad A=2.98 \times 10^{-4} \mu \Omega \mathrm{cm} / \mathrm{K}^{2}$ and $B$ $=1.1 \times 10^{-9} \mu \Omega \mathrm{cm} / \mathrm{K}^{9 / 2}$. The deduced value of the residual resistivity, $\rho_{0}$, is amongst the lowest values reported for $\mathrm{NiMnSb}$ to date. ${ }^{89}$ It is well known that a reliable indicator of sample purity is the residual resistivity ratio (RRR), i.e., the ratio of the resistivities at 300 and $0 \mathrm{~K}$. For the present sample RRR 3 which is amongst the highest values reported for NiMnSb. ${ }^{8,9}$ This value is still low compared to the values characterizing very pure samples, indicating the presence of a relatively large defect concentration in our samples. The $T^{9 / 2}$ term is attributed to two-magnon scattering, ${ }^{17}$ which is expected to occur in half metals for which electrons of only one spin direction exist at the Fermi level. The $T^{2}$ term, on the other hand, could be attributed either to one-magnon scattering or to electron-electron scattering that is present in a Fermi liquid. The presence of the $T^{9 / 2}$ term naturally constraints us to ascribe the $T^{2}$ term to the latter effect. It is noteworthy that the value of the prefactor, $A$, of the $T^{2}$ term falls within the accepted range of values for electronelectron scattering. In the main panel of Fig. 4, the contributions of the two $T$-dependent terms, $T^{2}$ and $T^{9 / 2}$, are shown separately.

An equally satisfactory fit of the resistivity data up to 70 $\mathrm{K}$ can also be obtained using the expression

$$
\rho(T)=\rho_{0}+A T^{2.5},
$$

with $\rho_{0}=8.6 \mu \Omega \mathrm{cm}$ and $A=4.15 \times 10^{-11} \mu \Omega \mathrm{cm} / \mathrm{K}^{2.5}$. Note that such a power law for the resistivity has been observed also in manganites. ${ }^{18,19}$ Auslender et al. ${ }^{18}$ associated the $T^{2.5}$ term with the scattering of holes by magnons without spin flip, whereas Schiffer et al. ${ }^{19}$ suggested the $T^{2.5}$ term as an empirical fit to the resistivity data representing a combination of electron-electron, electron-phonon, and electronmagnon scattering.

It is noteworthy to point out that another satisfactory fit of the resistivity data up to $70 \mathrm{~K}$ can also be obtained using the expression

$$
\rho(T)=\rho_{0}+A T^{2}+B T^{5},
$$

with $\rho_{0}=8.6 \mu \Omega \mathrm{cm}, A=3 \times 10^{-4} \mu \Omega \mathrm{cm} / \mathrm{K}^{2}$, and $B=1.2$ $\times 10^{-10} \mu \Omega \mathrm{cm} / \mathrm{K}^{5}$. In this expression the $T^{5}$ term is due to the electron-acoustic phonon scattering process. ${ }^{20}$ However, it is well known that the $T^{5}$ term is dominant at temperatures lower than $0.1 \Theta_{D} \sim 25-30 \mathrm{~K}^{21}$ (the Debye temperature, $\Theta_{D}$, of $\mathrm{NiMnSb}$ is estimated to be between 250 and $300 \mathrm{~K}){ }^{9}$ Based on this fact it is concluded that the equation containing the $T^{9 / 2}$ term is more appropriate for describing the present resistivity data.

At temperatures above $120 \mathrm{~K}$, the resistivity is proportional to $T^{1.55}$. Such a power law has not been associated with any particular scattering process. However, it is noteworthy to mention that the deduced exponent (1.55) is very close to the exponent $5 / 3$, which describes the temperature dependence of the resistivity of spin fluctuation magnets. ${ }^{22}$ We propose, therefore, that our high-temperature resistivity data indicate that the investigated sample behaves as an itinerant ferromagnet consistently with the observed hightemperature magnetization behavior mentioned earlier. Both the change in the temperature dependence of the resistivity 


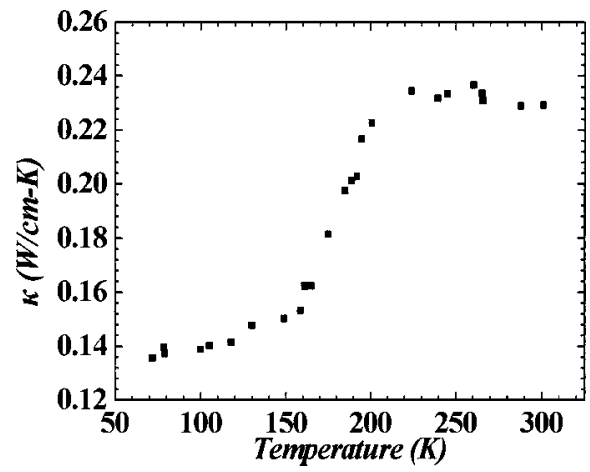

FIG. 5. Temperature dependence of the thermal conductivity of specimen No. 6 .

and the fact that value of the residual resistivity derived from fitting the high-temperature resistivity data $(T>120 \mathrm{~K})$ is higher than the value of the residual resistivity derived from fitting the low-temperature data $(T<70 \mathrm{~K})$, are consistent with the scenario that both spin bands contribute to transport above $\sim 100 \mathrm{~K}$ due to the opening of the spin-flip scattering channel at these temperatures. Therefore, the resistivity results are consistent with a crossover from a half-metallic-like behavior at low temperatures to a normal itinerant behavior at high temperatures.

\section{Thermal conductivity}

Figure 5 shows the temperature dependence of the thermal conductivity, $\kappa$, for specimen No. 6 in the temperature range $70-320 \mathrm{~K}$. In general, the total thermal conductivity of magnetic metals comprises an electronic $\left(\kappa_{e}\right)$, a lattice $\left(\kappa_{l}\right)$, and a magnon $\left(\kappa_{m}\right)$ contribution

$$
\kappa=\kappa_{e}+\kappa_{l}+\kappa_{m} .
$$

It is well known that for $T \geqslant \Theta_{D}$, the electronic contribution can be derived from the electrical resistivity, $\rho(T)$, using the Wiedemann-Franz law

$$
\kappa_{e}=\frac{L_{0} T}{\rho(T)},
$$

where $L_{0}$ is the Lorentz number. A comparison of $\kappa_{e}$ with $\kappa$ at room temperature indicates that the electronic contribution to thermal conductivity is the most dominant among the contributions outlined above $\left(\kappa_{e} / \kappa \sim 85 \%\right)$. (For this calculation we used the typical value of the Lorenz number $L_{0}$ $=2.45 \times 10^{-8} \mathrm{~W} \Omega / \mathrm{K}^{2}$ which is valid for $T>\Theta_{D}$. )

While above $200 \mathrm{~K} \kappa(T)$ is nearly constant, below 200 $\mathrm{K}$ it decreases linearly on cooling down to $150 \mathrm{~K}$ with a positive slope; this behavior is characteristic of intermetallics. ${ }^{23}$ However, below $150 \mathrm{~K}$ we observe that the thermal conductivity decreases with a slower rate. This change of slope may be taken as an indication that at temperatures lower than $150 \mathrm{~K}$ the scattering of the carriers is reduced. This effect in $\kappa(T)$ along with the features observed in the temperature dependence of both the magnetization and resistivity, are consistent with a possible crossover from halfmetallic behavior to normal ferromagnetic behavior in the temperature region $80-150 \mathrm{~K}$.

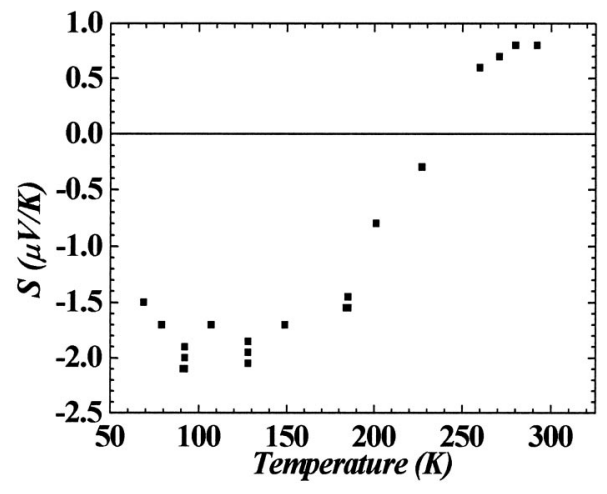

FIG. 6. Temperature dependence of the thermopower of specimen No. 6.

\section{Thermopower}

The temperature dependence of the Seebeck coefficient, $S(T)$, for specimen No. 6 is shown in Fig. 6. The most remarkable feature in the data is the change from a small negative to a small positive thermopower at $\sim 200 \mathrm{~K}$. The negative value of $S$ in the low temperature range indicates that the dominant entropy carriers at the Fermi surface are electrons whereas the positive value of $S$ above $200 \mathrm{~K}$ indicates that the dominant entropy carriers are holes. The sign change could be attributed either to the larger number of the thermally excited holes or due to the higher mobility of the contributing holes. The small absolute value of the Seebeck coefficient, $|S|<2 \mu \mathrm{V} / \mathrm{K}$, indicates that the two types of carriers are nearly compensated. The present temperature variation of the Seebeck coefficient agrees well with previously published data. ${ }^{9}$ It is noteworthy that the thermopower data in the range 70-200 K "disagree" with the low-field Hall effect data, which indicate that the dominant charge carriers are holes throughout the investigated temperature range ( 5 $<T<300 \mathrm{~K}){ }^{8,9}$ These seemingly contradictory results are probably caused by the complex Fermi surface of NiMnSb, which in addition to three anisotropic hole sheets contains electron-like parts as well as parts with open orbits. ${ }^{8}$

\section{Transport spin-polarization measurements-Andreev reflection}

The conductance spectra of nine different $\mathrm{Nb} / \mathrm{NiMnSb}$ (sample No. 6) contacts were measured and then fitted using the extended Blonder-Tinkham-Klapwijk model. ${ }^{24}$ This provided a value for the transport spin polarization, $P_{t}$, as a function of a dimensionless barrier parameter, $Z$ (the value of the superconducting energy of $\mathrm{Nb}$ was fixed to $1.5 \mathrm{meV}$ ). The plot of $P_{t}$ vs $Z$ for sample No. 6 is shown in Fig. 7. Note that for a clean interface $(Z=0)$ a value of $P_{t} \sim 45 \%$ has been measured. This much lower value of $P_{t}$ compared with the expected $100 \%$ spin polarization of the bulk, could be attributed to a number of reasons. Disorder at the surface, particularly antisite defects, is well known that result in a dramatic decrease in the spin polarization. Additionally, recent theoretical analysis has shown that both $\mathrm{Ni}$ - and $\mathrm{MnSb}$ terminated surface layers are not half metallic. ${ }^{25}$ Therefore, 


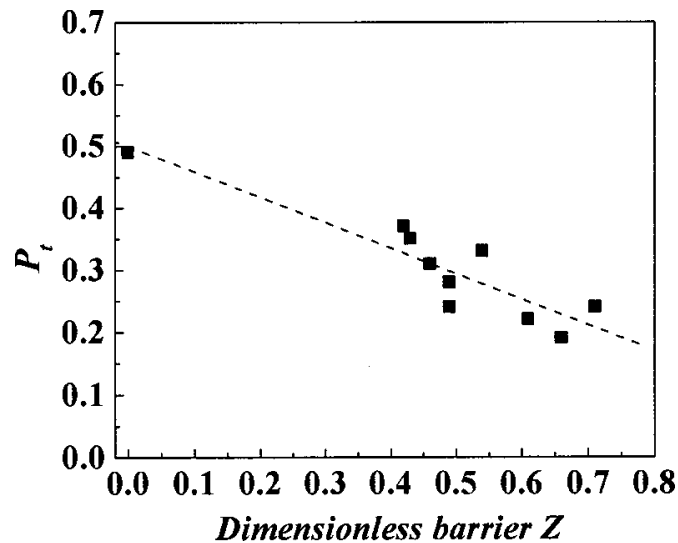

FIG. 7. Transport polarization vs dimensionless barrier parameter $(Z)$, for specimen No. 6.

the apparent disagreement between the indications coming from the bulk measurements for half-metallic behavior (i.e., $100 \%$ spin polarization) at low temperatures and the results of PCAR could be attributed to the fact that PCAR is a surface sensitive technique.

\section{CONCLUSIONS}

In summary, in this study we have identified the optimum conditions for synthesizing good quality NiMnSb bulk material using arc melting. All investigated physical properties indicate that in the temperature region $80-150 \mathrm{~K}$ a crossover most likely takes place from half metallic behavior to normal ferromagnetic behavior. Half metallicity in our $\mathrm{NiMnSb}$ specimen is well supported by the integer saturation magnetization value at $5 \mathrm{~K}$, the temperature dependence of the saturation magnetization which follows the $T^{3 / 2}$ Bloch law up to $200 \mathrm{~K}$, and the $T^{9 / 2}$ contribution in the lowtemperature resistivity implying two-magnon scattering (identified experimentally). On the other hand, PCAR, a surface sensitive technique, demonstrates only $45 \%$ spin polarization.

\section{ACKNOWLEDGMENTS}

The authors gratefully acknowledge support from the FENIKS Project No. (EC:G5RD-CT-2001-00535). The authors would also like to thank Dr. G. Kastrinakis for useful theoretical discussions.

${ }^{1}$ S. A. Wolf, D. D. Awschalom, R. A. Burham, J. M. Daughton, S. von Molnár, M. L. Roukes, A. Y. Chtchelkanova, and D. M. Treger, Science 294, 1488 (2001).

${ }^{2}$ S. Datta and B. Das, Appl. Phys. Lett. 56, 665 (1990).

${ }^{3}$ S. Gardelis, C. G. Smith, C. H. W. Barnes, E. H. Linfield, and D. A. Ritchie, Phys. Rev. B 60, 7764 (1999); C.-M. Hu, J. Nitta, A. Jensen, J. B. Hansen, and H. Takayanagi, ibid. 63, 125333 (2000).

${ }^{4}$ G. Schmidt, D. Ferrand, L. W. Molenkamp, A. T. Filip, and B. J. van Wees, Phys. Rev. B 62, R4790 (2000).

${ }^{5}$ R. A. de Groot, F. M. Mueller, P. G. van Engen, and K. H. J. Buschow, Phys. Rev. Lett. 50, 2024 (1983).

${ }^{6}$ M. J. Otto, H. Feil, R. A. M. van Woerden, J. Wijngaard, P. J. van der Valk, C. F. van Bruggen, and C. Haas, J. Magn. Magn. Mater. 70, 33 (1987).

${ }^{7}$ S. J. Youn and B. I. Min, Phys. Rev. B 51, 10436 (1995).

${ }^{8}$ M. J. Otto, R. A. M. van Woerden, P. J. van der Valk, J. Wijngaard, C. F. van Bruggen, C. Haas, and K. H. J. Buschow, J. Phys.: Condens. Matter 1, 2341 (1989); 1, 2351 (1989).

${ }^{9}$ C. Hordequin, D. Ristoiu, L. Ranno, and J. Pierre, Eur. Phys. J. B 16, 287 (2000).

${ }^{10}$ A. C. Rowe, Ph.D. thesis, Imperial College, London, 2000.; C. Grigorescu et al., in Supermaterials, NATO Series, edited by R. Cloots, M. Ausloos, R. Pekala, and G. Vacquier (Kluwer, Dordrecht, 2000), p. 173.

${ }^{11}$ A. Szytula, Ž. Dimitrijević, J. Todorović, A. Kołodziejczyk, J. Szelag, and A. Wanic, Phys. Status Solidi A 9, 97 (1972)

${ }^{12}$ R. J. Soulen et al., Science 282, 85 (1998).

${ }^{13}$ A. Lebail, H. Duroy, and J. L. Fourquet, Mater. Res. Bull. 23, 447 (1998).

${ }^{14}$ W. Marshall and S. Lovesey, Theory of Thermal Neutron Scattering (Oxford University Press, Oxford, 1971).

${ }^{15}$ I. Galanakis, P. H. Dederichs, and N. Papanikolaou, Phys. Rev. B 66, 134428 (2002).

${ }^{16}$ V. Irkhin and M. I. Katsnelson, J. Phys.: Condens. Matter 2, 7151 (1990).

${ }^{17}$ K. Kubo and N. A. Ohata, J. Phys. Soc. Jpn. 33, 21 (1972).

${ }^{18}$ M. Auslender, A. E. Kar'kin, E. Rozenberg, and G. Gorodetsky, J. Appl. Phys. 89, 6639 (2001)

${ }^{19}$ P. Schiffer, A. P. Ramirez, W. Bao, and S.-W. Cheong, Phys. Rev. Lett. 75, 3336 (1995).

${ }^{20}$ C. Kittel, in Introduction to Solid State Physics, 3rd ed. (Wiley, New York, 1967), p. 220

${ }^{21}$ J. Ziman, in Electrons and Phonons (Oxford, London, 1960), p. 365.

${ }^{22}$ K. Ueda and T. Moriya, J. Phys. Soc. Jpn. 39, 605 (1975).

${ }^{23}$ S. Y. Li et al., Phys. Rev. B 65, 064534 (2002).

${ }^{24}$ I. I. Mazin, A. A. Golubov, and B. Nadgorny, J. Appl. Phys. 89, 7576 (2001).

${ }^{25}$ G. A. de Wijs and R. A. de Groot, Phys. Rev. B 64, 020402 (2001). 